This item was submitted to Loughborough's Research Repository by the author.

Items in Figshare are protected by copyright, with all rights reserved, unless otherwise indicated.

\title{
Laparoscopic surgical skills training: an investigation of the potential of using surgeons' visual search behaviour as a performance indicator
}

PLEASE CITE THE PUBLISHED VERSION

http://dx.doi.org/10.1117/12.2044387

PUBLISHER

(c) SPIE

VERSION

VoR (Version of Record)

LICENCE

CC BY-NC-ND 4.0

\section{REPOSITORY RECORD}

Chen, Yan, Leng Dong, Alastair G. Gale, Benjamin Rees, and Charles Maxwell-Armstrong. 2019.

"Laparoscopic Surgical Skills Training: An Investigation of the Potential of Using Surgeons' Visual Search Behaviour as a Performance Indicator". figshare. https://hdl.handle.net/2134/19576. 


\title{
Laparoscopic surgical skills training: an investigation of the potential of using surgeons' visual search behaviour as a performance indicator
}

\author{
Yan Chen*a, Leng Dong, Alastair G. Gale ${ }^{\mathrm{a}}$, Benjamin Rees ${ }^{\mathrm{b}}$, \& Charles Maxwell-Armstrong $^{\mathrm{b}}$ \\ ${ }^{a}$ Applied Vision Research Centre, Loughborough University, Loughborough, UK; \\ ${ }^{\mathrm{b}}$ Queen's Medical Centre, Nottingham, UK
}

\begin{abstract}
Laparoscopic surgery is a difficult perceptual-motor task and effective and efficient training in the technique is important. Viewing previously recorded laparoscopic operations is a possible available training technique for surgeons to increase their knowledge of such minimal access surgery (MAS). It is not well known whether this is a useful technique, how effective it is or what effect it has on the surgeon watching the recorded video. As part of an on-going series of studies into laparoscopic surgery, an experiment was conducted to examine whether surgical skill level has an effect on the visual search behaviour of individuals of different surgical experience when they examine such imagery. Medically naive observers, medical students, junior surgeons and experienced surgeons viewed a laparoscopic recording of a recent operation. Initial examination of the recorded eye movement data indicated commonalities between all observers, largely irrespective of surgical experience. This, it is argued, is due to visual search in this situation largely being driven by the dynamic nature of the images. The data were then examined in terms of surgical steps and also in terms of interventions when differences were found related to surgical experience. Consequently, it is argued that monitoring the eye movements of trainee surgeons whilst they watch pre-recorded operations is a potential useful adjunct to existing training regimes.
\end{abstract}

Keywords: Minimal Access Surgery, eye movement, surgical performance

\section{INTRODUCTION}

Laparoscopic surgery, also known as minimally invasive surgery (MIS), or minimal access surgery (MAS), requires additional skills to the traditional open surgery which makes the domain of key interest to medical imaging researchers. In MAS the abdomen of the patient is distended with carbon dioxide gas and a co-aligned miniature camera and light source are inserted via a trocar. The surgeon operates by watching the resultant camera image of the patient's internal organs on a monitor, placed at the other side of the patient, and uses relevant laparoscopic instruments which are inserted, manipulated and removed, via two other trocars. The camera is operated by another person. Whilst the displayed camera image is typically representative of the surgeon's viewpoint with respect to the instruments s/he is using, this is not necessarily so. Therefore operating in this manner requires considerable visual skills as well as manual dexterity.

Training in open surgery traditionally has followed a master-apprentice model approach where the experienced surgeon supervises a trainee/junior surgeon as they carry out various procedures. Increasingly training approaches employing virtual reality (VR) simulators of differing degrees of complexity have become more commonplace. In MAS training using surgical simulators is particularly necessary as the surgeon needs considerable practice in performing operations whilst indirectly watching their instrument manipulations on a monitor. The need for a rigorous approach to training MAS skills has been pointed out ${ }^{1}$ as well as the need for a reliable method of skill assessment. Surgeons can also practice laparoscopic operations on human cadavers which have the advantages of providing appropriate tissue consistency, a realistic operating experience and the correct anatomy ${ }^{2}$. Yet another way of learning some aspects of MAS skills is to view previously recorded laparoscopic operations. As part of an on-going research project examining training and surgical performance in MAS surgery here we investigated the effect of differing surgical knowledge on where observers looked within the video image of pre-recorded MAS operations.

*y.chen@lboro.ac.uk

Medical Imaging 2014: Image Perception, Observer Performance, and Technology Assessment,

edited by Claudia R. Mello-Thoms, Matthew A. Kupinski, Proc. of SPIE Vol. 9037,903704

(C) 2014 SPIE · CCC code: $1605-7422 / 14 / \$ 18 \cdot$ doi: $10.1117 / 12.2044387$ 
Firstly a MAS procedure was carried out by a surgeon with the laparoscopic imagery recorded. We then monitored the search behaviour of a number of observers, of differing surgical knowledge level, as they viewed this recorded operation. It was hypothesised that surgical skill level would be related to eye movement parameters in a similar way as commonly found in radiological inspection tasks - namely with expertise being related to fewer eye fixations, longer fixations and longer saccades ${ }^{3}$. Surgically naive observers would be expected to exhibit fixation locations driven by the imagery whereas with increasing surgical knowledge it was expected that eye fixations would be related to the actual ongoing operation.

\section{METHODS}

A surgeon had previously performed a laparoscopic procedure, an appendicectomy (appendix removal), on a fresh human cadaver as part of a cadaveric laparoscopic training course. The display output from the laparoscopic camera was recorded (Figure 1).

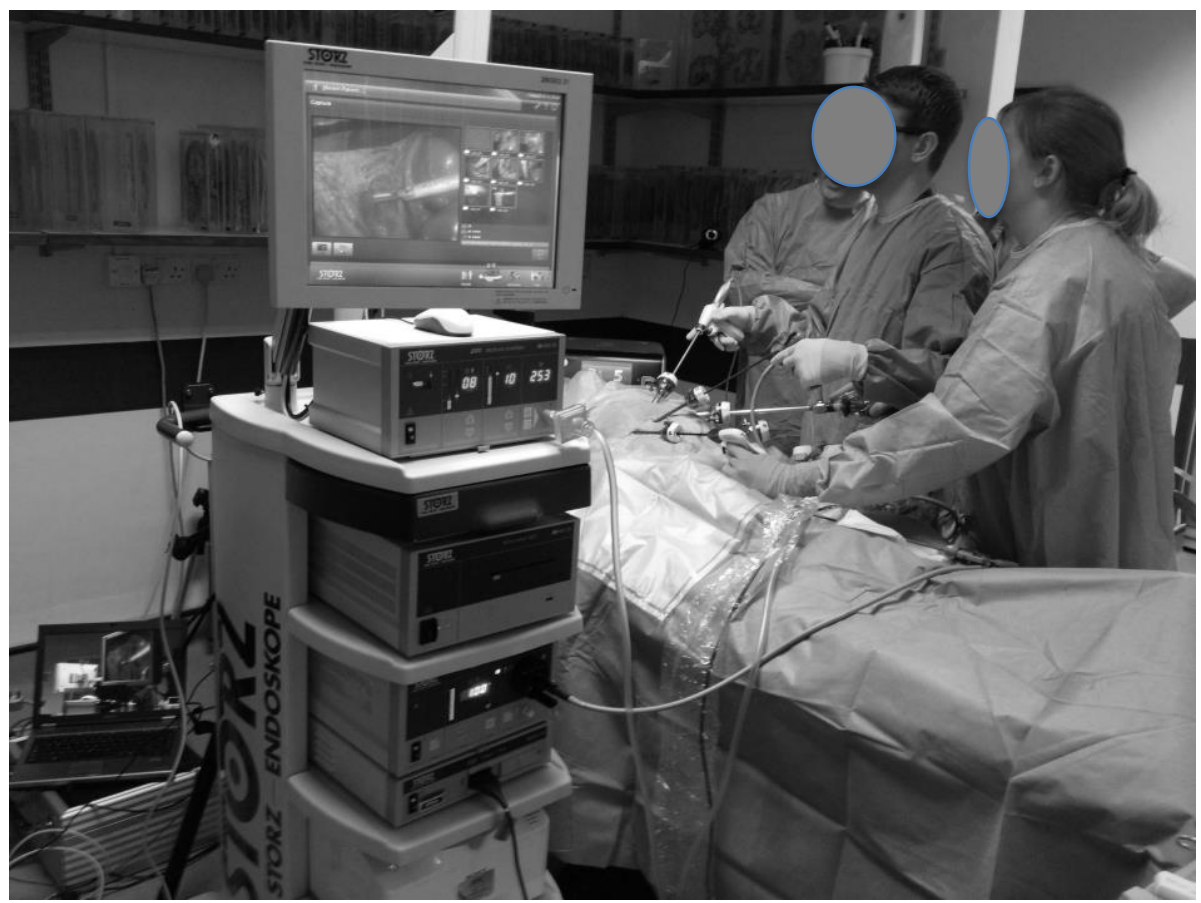

Figure 1. The operation in progress with the operating surgeon (centre) and colleagues watching the procedure on the main monitor which is located behind the slave monitor seen in the foreground. The individual in the foreground is operating the laparoscopic camera with the slave monitor showing the resultant camera scene.

The appendicectomy procedure took 40 minutes and 22 seconds. Afterwards, the surgical video was exported. As it was part of the training course, there were several parts of the video that were mainly training focused. The video was then edited to 11 minutes and 5 seconds by the operating surgeon so as to exclude the additional training information but keeping the key surgical steps of the procedure. Subsequently, the video of the operation was replayed individually to three groups of participants: ten surgically-naive computer science students, nine medical students and three experienced laparoscopic surgeons (taken here as 'experts'). The videos were shown on a 22-inch display (1,680 x 1,050 resolution) while their search behaviour was recorded using a Tobii X50 device (Figure 2).

The eye movement gaze point data were sampled at $50 \mathrm{~Hz}$. To examine differences between the groups in these data samples, the data timestamps were matched for each participant's raw eye movement data. The sample data of one expert surgeon (who had the longest surgical experience) was taken as a standard and each participant's gaze data were then 
compared with this expert's sample data on a frame by frame basis and the mean distance (pixels) between the expert's and participants' gaze point on each sample was calculated.

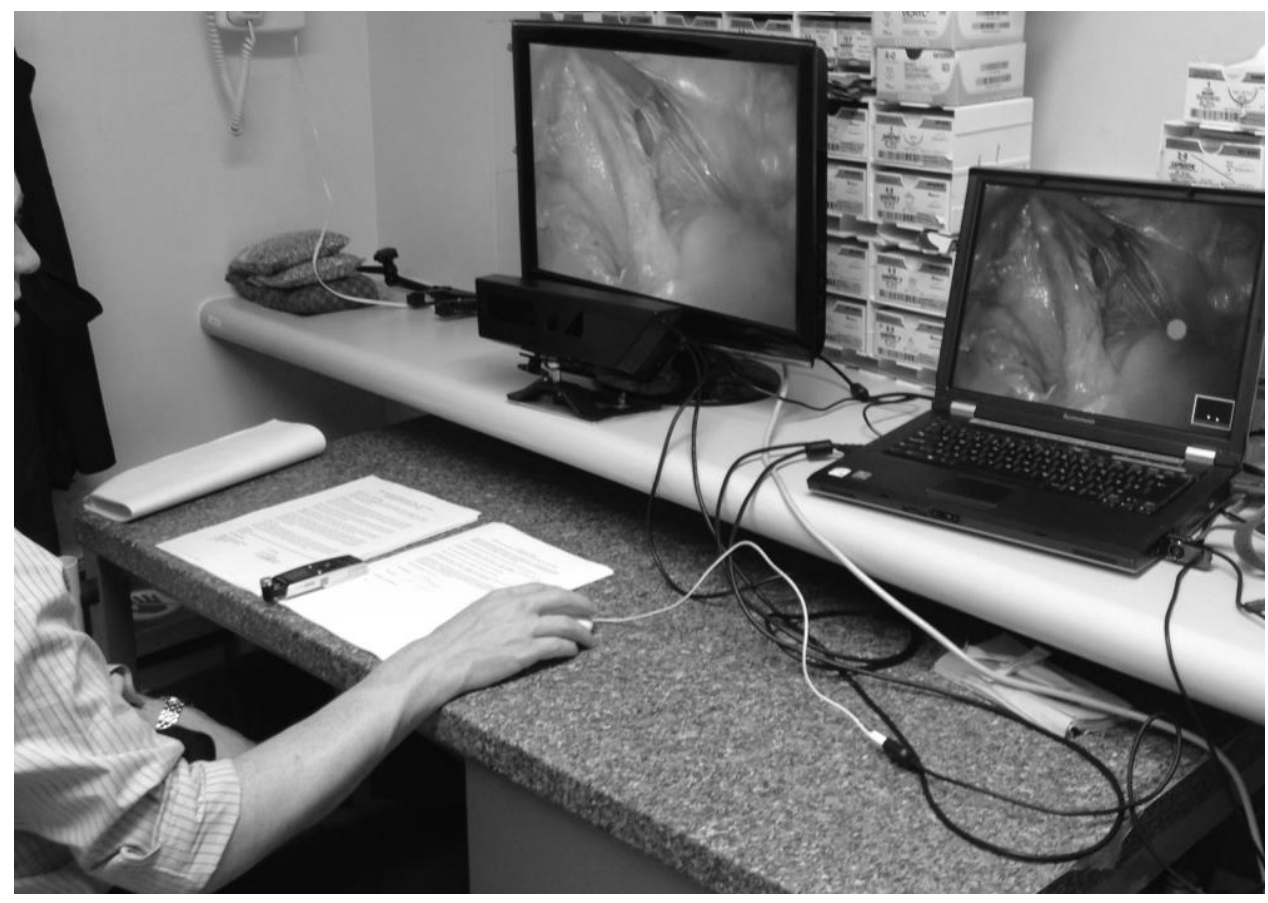

Figure 2. Participant watching the surgical video while their eye movements are recorded.

To perform more detailed analyses the video records were segmented into various surgical steps as specified by the Procedure Based Assessment (PBA) by the Intercollegiate Surgical Curriculum Programme (ISCP). There are six key operational steps in such an operation:

1. Performs exploration of the right iliac fossa in a logical fashion

2. Mobilises appendix safely

3. Achieves secure haemostasis of mesoappendix then divides this safely

4. Divides the appendix safely with appendix stump secured

5. Examines the omentum, terminal ileum and pelvic organs when the appendix is found to be macroscopically normal

6. Manages intraperitoneal contamination at end of procedure appropriately

Of importance here are steps 2, 3 and 4. The operating surgeon identified the relevant points on the video stream when these steps began and ended. The pertinent eye movement data of these three steps were then examined by calculating the average distance between the expert and participants' gaze point location on each frame (pixels per frame).

Lastly, the eye movement data were examined whenever a discrete change took place in the recorded image stream; such as when a new surgical instrument was inserted/removed or the video edited to the next part of the operation, in order to determine whether, and how quickly, participants fixated on the new surgical target. There were 14 such interventions. Data were examined from all 22 participants in the five seconds before and after these interventions yielding 616 sets of data.

\section{RESULTS}

It was shown that participants with more medical knowledge looked closer to where the expert looked (Figure 3 and Table 1), although, one-way ANOVA found no significant difference between experience groups $(\mathrm{F}(2,18)=1.229, p=$ 
n.s., $r=.35)$. Post hoc t-tests found no significant difference either between the novices group and medical student group ( $p=$ n.s.) or between the novices group and the expert group ( $p=$ n.s.). A contrast test was performed between a combined group with medical knowledge (i.e. experts and medical students group) and the non-medical knowledge group (i.e. novices) which was not significant ( $\mathrm{t}(18)=-1.26, p=$ n.s., $r=.308)$.

\begin{tabular}{l|l}
\hline Experience group & Average distance (Pixels/sample) \\
Novices & 107.4370865 \\
Medical Student & 101.3295191 \\
Expert & 93.57756721 \\
\hline
\end{tabular}

Table 1 The average distance (pixels/sample) between each participant's eye gaze point and the standard gaze location by experience group

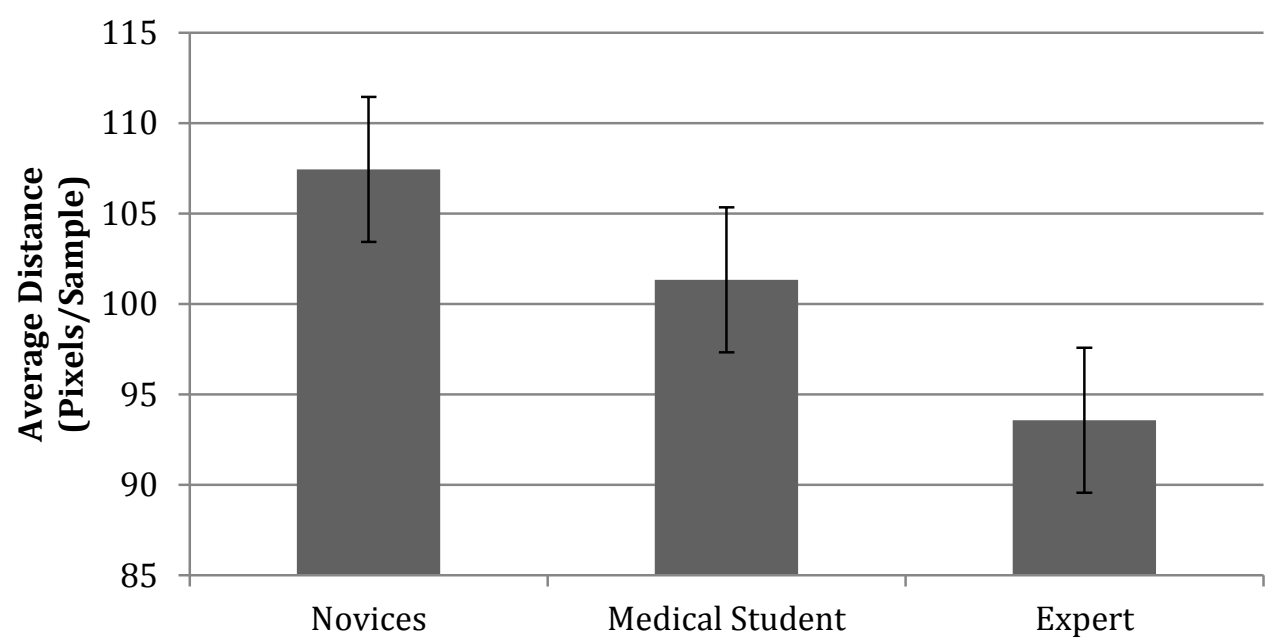

Figure 3. Comparison of the average distance (pixels/sample) of each participant's eye gaze point and the standard location between experience groups

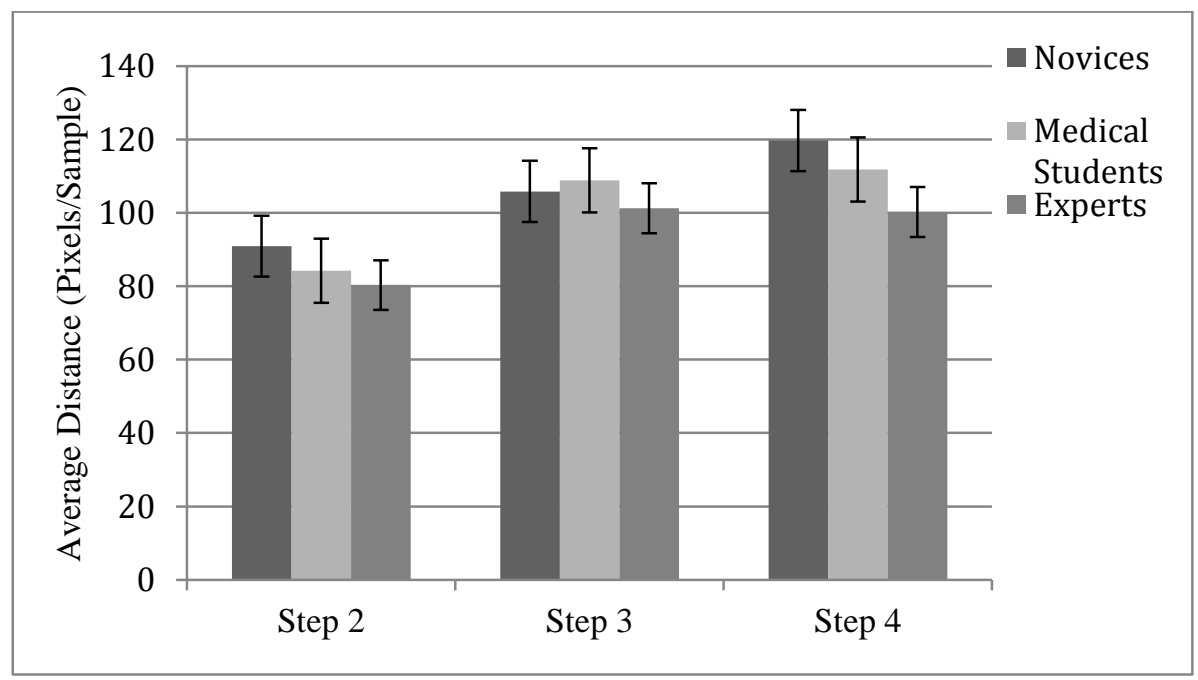

Figure 4. The average distance (pixels/sample) in each experience group per surgical steps 
In order to verify this finding then the gaze location of another expert from the expert group was set to be the standard and the same data analyses performed. A similar result was found - participants with more medical knowledge looked in similar areas to the expert surgeon although no significant differences were found.

Surgical steps analysis

The surgical steps data are shown in Figure 4. For each step, the experienced surgeons viewed more similarly to the expert surgeon. One-way ANOVA was carried out comparing between groups on each surgical step. There was no significant difference found between the experience groups for any step examined (Step 2: $\mathrm{F}(2,18)=1.366, p=\mathrm{n}$.s., $r=.39$; Step 3: $\mathrm{F}(2,18)=0.190, p=$ n.s., $r=.21$; Step 4: $\mathrm{F}(2,18)=1.288, p=$ n.s., $r=.35)$. A contrast test was also performed between a group with medical knowledge (experts and medical students group combined) and non-medical knowledge group but found no significant difference (Step 2: $\mathrm{t}(18)=-1.61, p=\mathrm{n}$. .s., $r=.40$; Step 3: $\mathrm{t}(18)=-0.96, p=n . s ., r=.23$; Step 4: $\mathrm{t}(18)=-$ $1.61, p=$ n.s., $r=.40)$.

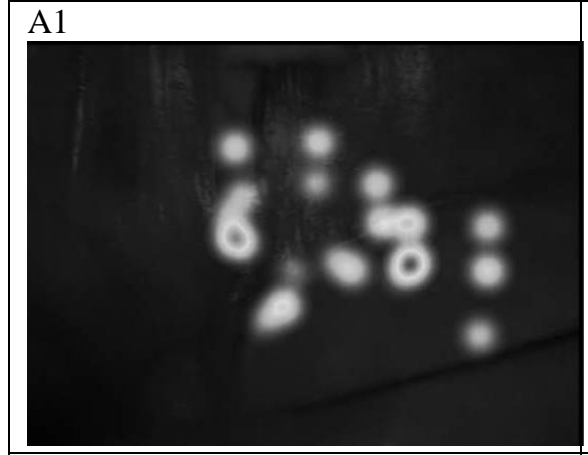

B1
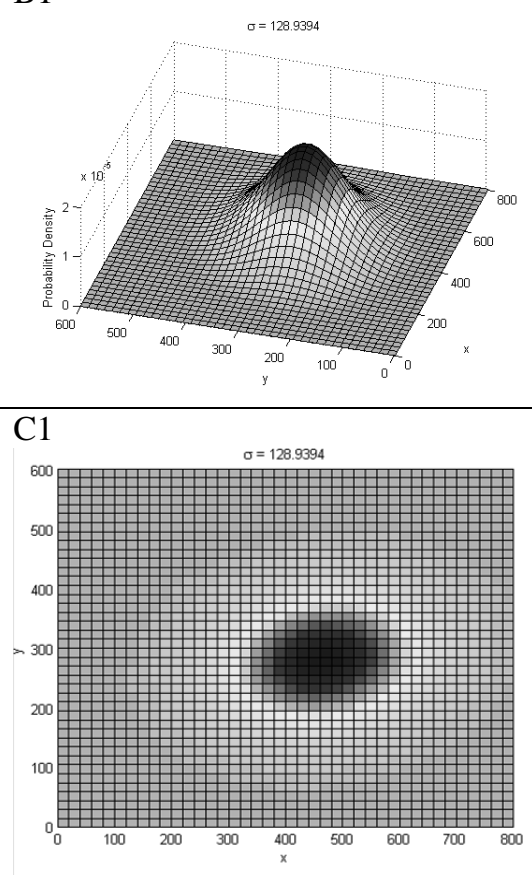

A2

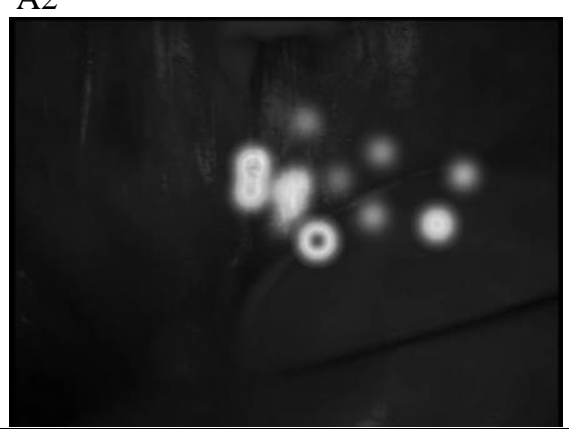

B2

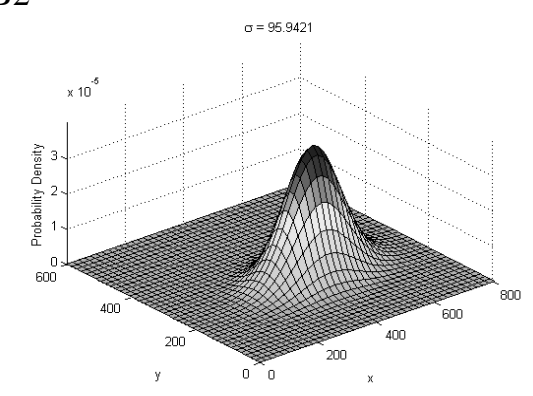

$\mathrm{C} 2$

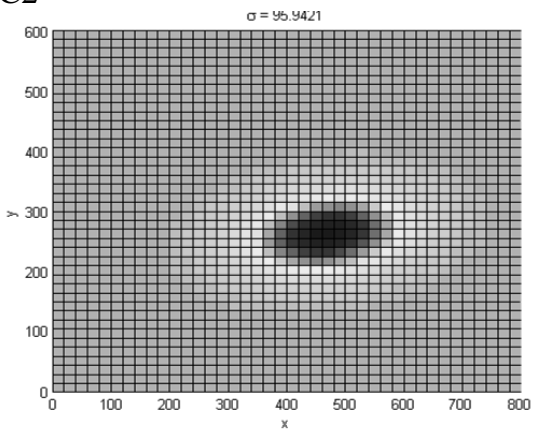

A3

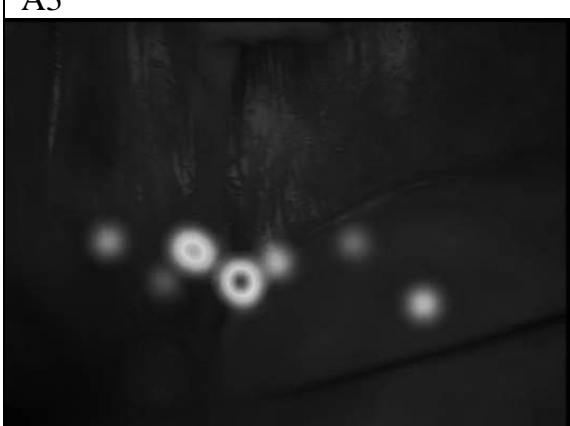

B3
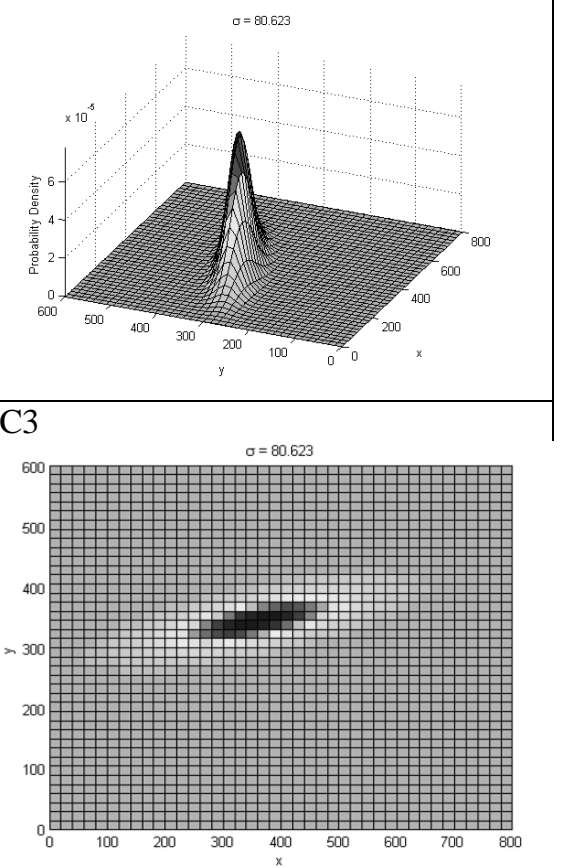

Figure 5. For explanation see text 
Intervention analysis

Figure 5 shows an example of the gaze location distribution heat map ( 5 seconds) by an expert, a medical student and a novice after the same intervention; this is shown as $\mathrm{x}-\mathrm{y}$; normalised 3D and 2D plots. The average distance to the spatial gaze location distribution in the example are $\sigma=128.94$ for experts, $\sigma=95.94$ for medical students and $\sigma=80.62$ for novices. This figure shows: an example of the expert's gaze location distribution (A1), a medical student's gaze location distribution (A2) and a novice's gaze location distribution (A3) after the same intervention. The same data shown as a normalised 3D distribution of the expert's gaze location distribution (B1), the medical student's gaze location distribution (B2) and normalised 3D distribution of the novice's gaze location distribution (B3). 2D heat maps of the distribution of the expert's gaze location distribution $(\mathrm{C} 1)$, the medical student's gaze location distribution $(\mathrm{C} 2)$ and 2D heat map of the novice's gaze location distribution (C3) for this example are also shown.

For each participant, both the mean value of the average spatial gaze location distribution before and after the intervention for all 14 interventions were calculated and compared (Table 2).

\begin{tabular}{llll}
\hline & $\sigma$ Experts & $\sigma$ Medical Students & $\sigma$ Novices \\
5 s before & 79.45137158 & 86.60518125 & 83.78080115 \\
5 s after & 89.79609794 & 90.2761264 & 80.01727614 \\
\hline
\end{tabular}

Table 2. The average distance $(\sigma)$ of each experience group before ( 5 seconds) and after (5seconds) the interventions.

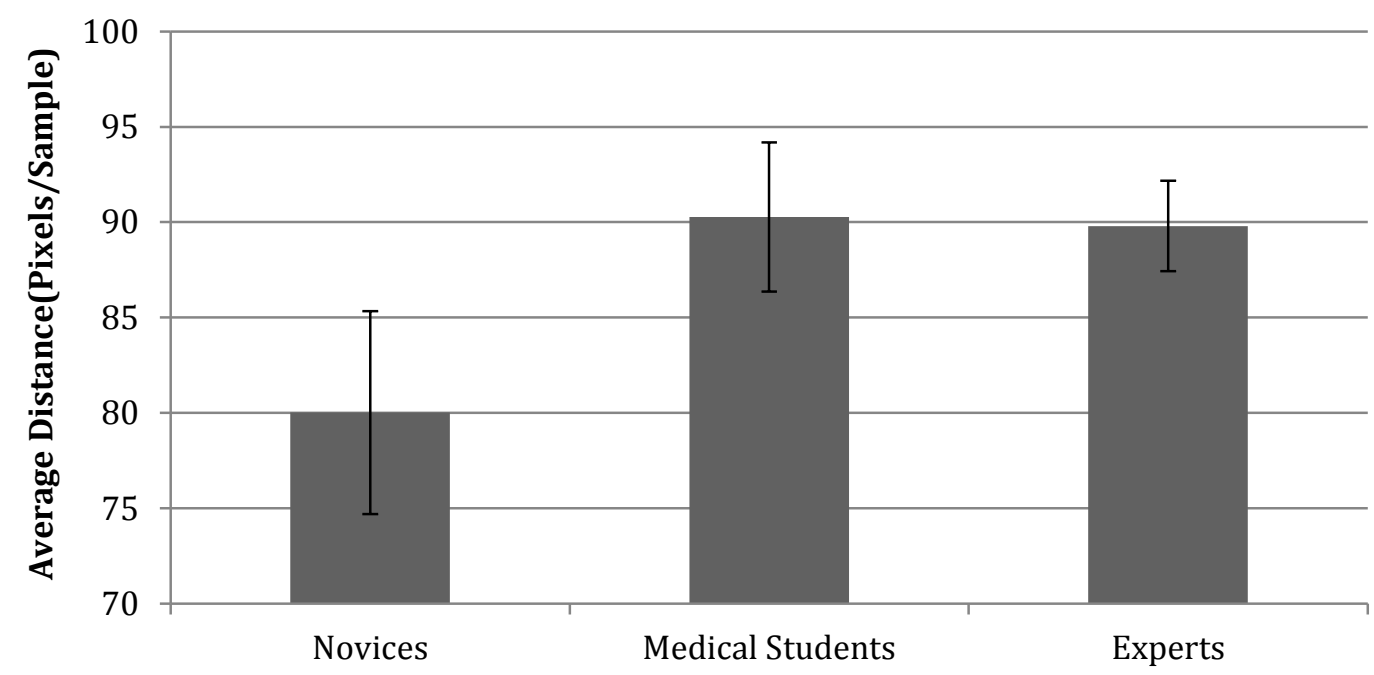

Figure 6. The average distance $(\sigma)$ of each experience group between the real time gaze location and the centre of spatial gaze location distribution (5 seconds after the intervention) 


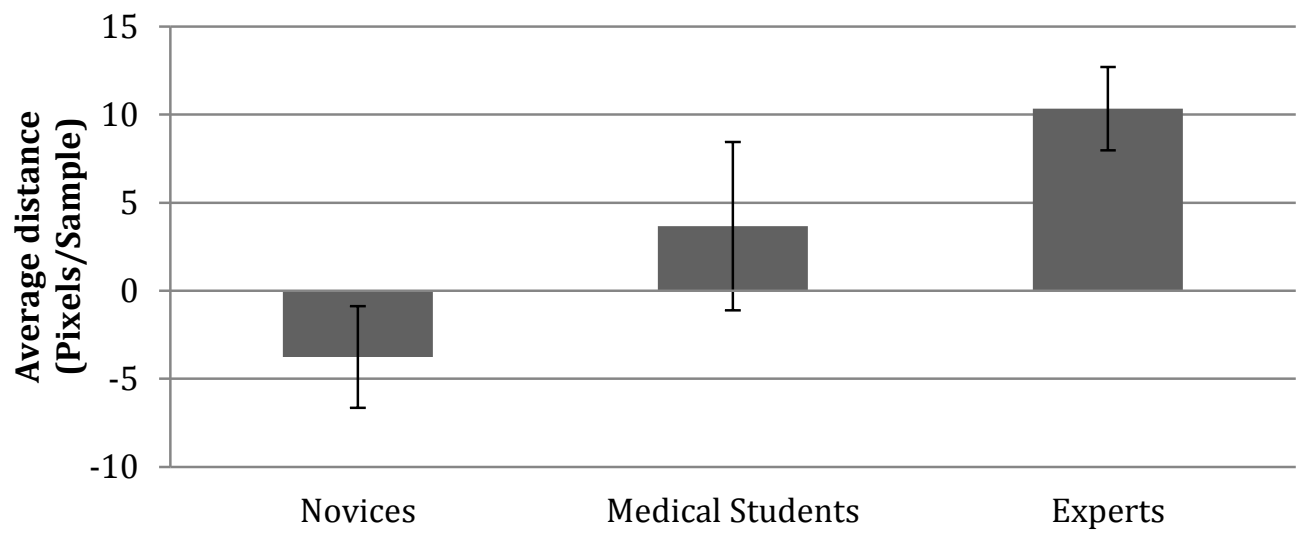

Figure 7. Difference between the average distance between the real time gaze location and the centre of spatial gaze location distribution ( 5 seconds after the intervention) and the average distance between the real time gaze location and the centre of spatial gaze location distribution ( 5 seconds before the intervention).

As seen in figure 6 , for the 5 seconds after the intervention, the experts and medical students visually examined more of the display than did the naive students. Although one-way ANOVA did not show any significant difference between experience groups $(\mathrm{F}(2,19)=0.265, p=n . s ., r=.47)$, a contrast test showed a significant difference between a grouping with medical knowledge (i.e. experts and medical students combined) and the group without medical knowledge (i.e. novices) $(\mathrm{t}(19)=2.138, p<.05, r=.47)$. Additionally, a post hoc $\mathrm{t}$-test indicated that the medical students average distance from the gaze centre were significantly higher than that of novices $(p<.05)$. However, no significant difference was found between experts and novices $(p=n . s$.), presumably due to the low number of participants in the expert group.

Figure 7 shows the difference per group of the average spatial distribution of gaze points between the 5 seconds before and 5 seconds after all interventions. This shows that after an intervention the novices essentially 'stared' at the display as compared to the other two groups. Medical students explored the new imagery and the experts explored the new imagery much more. Hence medical knowledge was related to image exploration.

\section{DISCUSSIONS}

The visual search behaviour of surgeons has previously been recorded during tasks such as simulated laparoscopic surgery ${ }^{4}$ demonstrating differences between experienced surgeons and novices ${ }^{5,6}$. For instance with the experienced spending more time fixating targets than novices ${ }^{6,7}$. Elsewhere, previous work has shown that surgical operative skill can be evaluated reliably by raters watching previously recorded laparoscopic operations ${ }^{8}$. Here we examined individuals of differing surgical/anatomical knowledge and how where they fixated on the laparoscopic imagery varies. It was found that with experience individuals fixated in locations more akin to the fixation locations of an expert surgeon. Breaking the task into surgical steps was thought would serve to distinguish between the experience groups easily but this was not found. Examining the data in terms of distinct visual interventions in the laparoscopic imagery then experts appeared to search the image display more - this inherently seems to be in contrast to the results of expertise in static medical images.

Clearly distinguishing between naive and experienced in this task was more troublesome than often found in typical 2D medical imaging tasks. The accepted interpretation of this experienced skilled visual behaviour is interpreted as demonstrating that with experience experts know where 'best' to look in an image type with which they are familiar and therefore fixate in high probability areas of an abnormality. Given the very different type of imaging involved here which is generally devoid of a specific single small prescribed target (as in many medical imaging tasks which are commonly researched) then such behaviour may or may not be expected. This further demonstrates the need for further 
consideration of the type of eye movement measures investigated with such images as has previously been argues with the dynamic images produced during CT Colonoscopy ${ }^{9}$.

\section{CONCLUSION}

A potential form of laparoscopic surgical training is simply to watch recordings of previous surgical procedures. It can be difficult to assess whether this is indeed useful or how this affects the surgeon's developing surgical skill level. The current work suggests that by breaking the recorded surgical task into segments and monitoring the eye movements of the trainee surgeon then a better indication of their current skill level can be obtained. This is our first examination of eye movement surgical data and only initial data treatments are presented here. More detailed analyses are ongoing as well as further experimental studies.

\section{References}

[1] Hamdorf J.M. \& Hall J.C., Acquiring surgical skills. Br J Surg. 2000 Jan;87(1):28-37.

[2] Lloyd, G.M., Maxwell-Armstrong, C., Acheson A.G., Fresh frozen cadavers: an underutilized resource in laparoscopic colorectal training in the United Kingdom, Colorectal Disease, 2011, Vol 13 (9), e303-e304

[3] Gale A.G., Human response to visual stimuli. In Hendee W. \& Wells P. (Eds.) Perception of Visual Information second edition, (New York) Springer Verlag, 1997.

[4] Tien G., Zheng B., \& Swindells C., Measuring situation awareness of surgeons in laparoscopic training, Proceedings of the 2010 Symposium on Eye-Tracking Research \& Applications, ACM, New York, 2010, 149-152

[5] Law B., Atkins M.S., Kirkpatrick A.E., \& Lomax A.J., Eye gaze patterns differentiate novice and experts in a virtual laparoscopic surgery training environment. Proceedings of the 2004 symposium on Eye tracking research \& applications, ACM, New York, 2004, 41-48.

[6] Wilson M., McGrath J., Vine S., Brewer J., Defriand D. \& Masters R., Psychomotor control in a virtual laparoscopic surgery training environment: gaze parameters differentiate novices from experts. Surg. Endosc., 2010, 24, 2458-2464.

[7] Wilson M.R., Vine S.J., Bright E., Masters R.S.W., Defriand D. \& McGrath J.S., Gaze training enhances laparoscopic technical skill acquisition and multi-tasking performance: a randomized controlled trial, Surg. Endosc. 2011, 25, 3731-3739

[8] Vassiliou MC, Feldman LS, Fraser SA, Charlebois P, Chaudhury P, Stanbridge DD, \& Fried GM. Evaluating intraoperative laparoscopic skill: direct observation versus blinded videotaped performances. Surg. Innov. 2007 Sep;14(3):211-6.

[9] Phillips, P., Boone, D., Mallet, S., Taylor, S., Altman, D., Manning, D., Gale, A., Halligan, S. Tracking eye gaze during interpretation of endoluminal 3D CT Colonography: Technical description and proposed metrics for analysis. Radiology 2013 Jun; 267(3):924-31. 